\title{
Differential microRNA expression profile comparison between epidermal stem cells and differentiated keratinocytes
}

\author{
ZHIFANG SONG ${ }^{1}$, DEWU LIU ${ }^{2}$, YAN PENG $^{2}, \mathrm{JIN} \mathrm{LI}^{2}$, ZHIWEI ZHANG ${ }^{2}$ and PU NING ${ }^{2}$ \\ ${ }^{1}$ Department of Cardiovascular Medicine and ${ }^{2}$ Burn Center, The First Affiliated Hospital of Nanchang University, \\ Nanchang, Jiangxi 330006, P.R. China
}

Received December 8, 2013; Accepted September 12, 2014

DOI: $10.3892 / \mathrm{mmr} .2014 .2886$

\begin{abstract}
The aim of the current study was to analyze the differential microRNA (miRNA) expression profiles of human epidermal stem cells (ESCs) and differentiated keratinocytes. Enzyme digestion was used in combination with rapid adhesion to collagen IV to isolate primary human ESCs and differentiated keratinocytes, from which total RNA was extracted. Fluorescence labeling, microarray hybridization and differential expression analyses were performed. Reverse transcription quantitative polymerase chain reaction (RT-qPCR) was performed to validate the reliability of the microarray results and predict the target genes of the differentially expressed miRNAs. A total of 25 miRNAs, including hsa-miR-197-5p, hsa-miR-125b-5p and hsa-miR-376a-3p, were upregulated, whereas 166 miRNAs, including hsa-miR-29b-3p, hsa-miR-203 and hsa-miR-34a-3p, were downregulated in the human ESCs compared with the expression in differentiated keratinocytes. RT-qPCR results confirmed the upregulation of hsa-miR-197-5p and the downregulation of hsa-miR-29b-3p, which were consistent with the microarray results. miRNA target prediction indicated that the miRNA expression levels correlated with cell proliferation, differentiation, apoptosis and senescence. Expression levels of miRNAs significantly differed between human ESCs and differentiated keratinocytes. This finding may be attributed to their biological characteristics, such as proliferative behavior and differentiation abilities.
\end{abstract}

\section{Introduction}

The skin acts as a defensive barrier, protecting the body against the external environment. Important physiological functions of the skin include protection, excretion, temperature

Correspondence to: Mr. Dewu Liu, Burn Center, The First Affiliated Hospital of Nanchang University, 17 Yongwaizheng Street, Nanchang, Jiangxi 330006, P.R. China

E-mail: dewuliucn@163.com

Key words: epidermal stem cells, keratinocytes, microRNA, gene expression profile regulation and stabilization of the internal environment (1). The epidermis is a stratified epithelium in the outer layer of the skin, where differentiated keratinocytes are constantly shed and replaced by new cells (2). Keratinocytes cannot divide, and their replacement depends on epidermal stem cells (ESCs), specialized stem cells of the skin that are located in the basal layer of the epidermis. ESCs have the potential for proliferation and differentiation and are the key seed cells for skin formation, repair and reconstruction (3).

MicroRNAs (miRNAs), non-coding RNA molecules of 19-22 nucleotides in length, have functions in the post-transcriptional regulation of the expression levels of genes or proteins (4). miRNAs participate in a series of important life processes, and are important molecules in cell proliferation, development and differentiation (5). Previous studies have confirmed that the deletion of the Dicer or DiGeorge syndrome critical region 8 , required in miRNA biosynthesis in neonatal mouse skin, causes defective skin barrier function, follicular dysplasia and excessive proliferation of epidermal basal cells $(6,7)$. This indicates that miRNA is essential for skin development and functional maintenance. However, the differential miRNA expression of epidermal cells at the various stages of differentiation remains unclear. Therefore, the present study used differential miRNA expression analysis to investigate the function and possible mechanisms of miRNAs in ESC proliferation and differentiation. The analysis was conducted using human ESCs and differentiated keratinocytes. The aims of this study were to elucidate the process and molecular pathways of normal skin development, providing evidence for future wound-healing gene therapy and a theoretical foundation for the clinical application of miRNAs.

\section{Materials and methods}

Sample collection. Prepuce samples were obtained from nine male patients aged 18-40 years old who underwent circumcision in the Department of Urology Surgery of the First Affiliated Hospital of Nanchang University (Nanchang, China) between January 2013 and April 2013. This study was conducted in accordance with the Declaration of Helsinki, and with approval from the Ethics Committee of Nanchang University. Written informed consent was obtained from all participants. 
Table I. Primer sequences in reverse transcription polymerase chain reaction.

miRNAs and primers

hsa-miR-197-5p

hsa-miR-197-5p RT primers

hsa-miR-197-5p antisense primer

hsa-miR-29b-3p

hsa-miR-29b-3p RT primers

hsa-miR-29b-3p antisense primer

U6 forward primer

U6 reverse primer

miRNA general sense
Primer sequence

CGGGTAGAGAGGGCAGTGGGAGG

GTCGTATCCAGTGCAGGGTCCGAG

GTATTCGCACTGGATACGACCCTCCC

TATATACGGGTAGAGAG GGCAGTG

TAGCACCATTTGAAATCAGTGTT

GTCGTATCCAGTGCAGGGTCCGA

GGTATTCGCACTGGATACGACAACACT

ACGATAGCACCATTTGA AATCAGT

CTCGCTTCGGCAGCACA

AACGCTTCACGAATTTGCGT

GTGCAGGGTCCGAGGT
Cell culture and identification. The epidermis was isolated with trypsin (Gibco-BRL, Carlsbad, CA, USA). Rapid adhesion to collagen IV (Sigma Aldrich, St. Louis, MO, USA) was used to isolate human ESCs and differentiated keratinocytes as previously described (8). The two cell groups were cultured in vitro in keratinocyte serum-free medium supplemented with epidermal growth factor and fetal bovine serum (Gibco-BRL). The cellular morphology of the two groups was observed under an inverted phase contrast microscope (CK40; Olympus, Japan). Immunostaining of integrins $\beta 1$, CK19, CK1 and CK10 (Abgent Inc., San Diego, CA, USA) was used for cell identification and performed according to the manufacturer's instructions.

Extraction of total RNA. Total RNA was extracted from the two cell groups with TRIzol (Invitrogen Life Technologies, Carlsbad, CA, USA). A spectrophotometer (ND-1000; NanoDrop, Wilmington, DE, USA) was used for quantification. Denaturing formaldehyde gel electrophoresis was performed to determine the total RNA quality. Purification of total RNA from the two groups was conducted using mirVana ${ }^{\mathrm{TM}}$ miRNA Isolation kit (Ambion Life Technologies, Carlsbad, CA, USA), followed by quantification. HeLa cell RNA (CapitalBio, Beijing, China) was used for positive control RNA

Fluorescence labeling and microarray hybridization. Total RNA (200 ng) from purified ESCs and differentiated keratinocytes were separately labeled with $\mathrm{Cy} 3$ and hybridized with Human miRNA Microarray Release 14.0, 8x15 K (Agilent, San Diego, CA, USA) for $20 \mathrm{~h}$ in a Hybaid thermal cycler (Peltier Thermal Cycler PTC-225; MJ Research, Waltham, MA, USA). The obtained RNA was washed with Gene Expression Wash Buffer (Agilent) for $5 \mathrm{~min}$ at room temperature and $5 \mathrm{~min}$ at $37^{\circ} \mathrm{C}$, and the microarray was subsequently scanned using an Agilent Microarray Scanner (G2565CA; Agilent).

Data extraction and analysis. Agilent Feature Extraction software v10.7 (Agilent) was used to analyze and extract data from the microarray image, and the data were normalized with the Agilent GeneSpring software GX10.0 (Agilent). Differential miRNA expression between the two cell groups was analyzed using Student's t-test. The screening condition was more than twice the difference in miRNA expression between ESCs and differentiated keratinocytes.

Reverse transcription quantitative polymerase chain reaction $(R T-q P C R)$ and miRNA target prediction. According to the microarray results, the expression levels of hsa-miR-197-5p were upregulated and the expression levels of hsa-miR-29b-3p, were downregulated, exhibiting strong original signals and clear differences, hence, these were selected for RT-qPCR verification. Briefly, $100 \mathrm{ng}$ of total RNA was reverse transcribed into cDNA as a template for qPCR amplification. The amplification conditions were as follows: $10 \mathrm{pmol} / 1$ of primer, denaturation at $95^{\circ} \mathrm{C}$ for $10 \mathrm{~min}$, followed by 40 cycles of $95^{\circ} \mathrm{C}$ for $15 \mathrm{sec}$ and $60^{\circ} \mathrm{C}$ for $1 \mathrm{~min}$. The melting curve was drawn at $60^{\circ} \mathrm{C}$ to $95^{\circ} \mathrm{C}$, and non-denaturing agarose gel electrophoresis of the $\mathrm{qPCR}$ products was performed (Applied Biosystems, Foster City, CA, USA). The primers (synthesized by Invitrogen Life Technologies) are shown in Table I. Expression levels were calculated using the comparative threshold cycle (CT) method. For the data analysis, the comparative CT value for U6 was used to normalize loading variations in the RT-qPCRs. The $\Delta \Delta \mathrm{Ct}$ of each sample was obtained by subtracting the $\Delta \mathrm{Ct}$ value of the sample from the $\Delta \mathrm{Ct}$ value of the reference. The $\Delta \Delta \mathrm{Ct}$ values were compared with the control by raising 2 to the $\Delta \Delta \mathrm{Ct}$ power. The RT-qPCR was performed in triplicate for each treatment group. TargetScan 6.2 software (http://www.targetscan.org) was used to predict the target genes of the differentially expressed miRNAs.

\section{Results}

Biological characteristics of the cells. The isolated cells were observed under a microscope. Fig. 1A shows uniformly distributed small round cells rapidly adhering to collagen IV with strong refractivity. Following three days of culture, these cells were firmly attached to the well and clones were clearly 


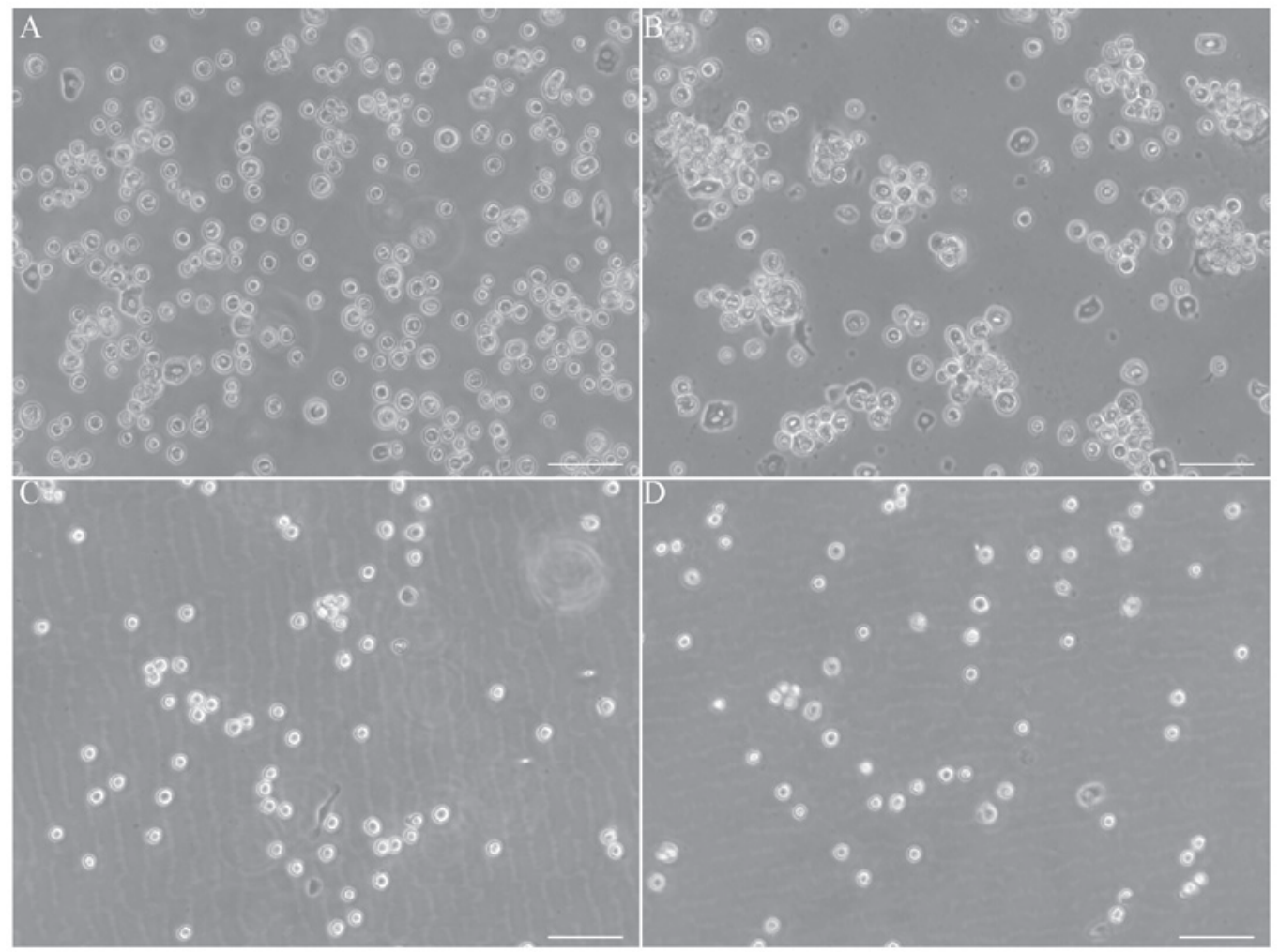

Figure 1. Primary human epidermal stem cells (ESCs) and differentiated keratinocytes (magnification, $\mathrm{x} 400$; scale bar, $20 \mu \mathrm{m}$ ). (A) Primary human ESCs screened by rapid adhesion to collagen IV. (B) Human ESCs in clonal growth following three days of cell culture. (C) Screened differentiated keratinocytes. (D) Differentiated keratinocytes without clones following three days of cell culture.

visible, which is in accordance with the characteristics of ESCs (Fig. 1B). Non-adherent cells were irregular in shape, size and distribution (Fig. 1C), loosely attached to the well and without clonal cells following 3 days of culture. This finding is in accordance with the characteristics of keratinocytes (Fig. 1D). Immunocytochemistry showed positive expression of CK19 and integrin $\beta 1$ in rapidly adherent cells, and CK1 and CK10 in non-adherent cells. These results are consistent with the characteristics of ESCs (Fig. 2A and B) and keratinocytes (Fig. 2C and D).

Quality control of total RNA. Total RNA was extracted from the human ESCs and differentiated keratinocytes (4.61 and $3.49 \mu \mathrm{g}$, respectively). The $18 \mathrm{~S}$ and $28 \mathrm{~S}$ rRNA bands were clearly visible in the RNA sample with an intensity ratio of $\geq 2: 1$, which is in accordance with the requirements of chip expression profiling (Fig. 3).

miRNA detection. Compared with differentiated keratinocytes, ESCs had 25 and 166 miRNAs with significant upregulation and downregulation, respectively. Tables II and III show miRNAs with $>5$-fold upregulation and downregulation, respectively. The miRNAs with the greatest upregulation and downregulation were hsa-miR-197-5p (21.98547 times) and hsa-miR-203 (25.45382 times), respectively.

$R T-q P C R$. Differentiated keratinocytes were used as a control. The $2^{-\Delta \Delta \mathrm{Ct}}$ value of two pairs of miRNAs was calculated according to the relative quantitative method (9). The $2^{-\Delta \Delta C t}$ values indicated upregulation of hsa-miR-197-5p
(13.830) and downregulation of hsa-miR-29b-3p (0.208), which is consistent with the microarray results.

Predicting target genes. Screened target genes showed close correlation between their biological function and cell proliferation, differentiation, apoptosis, senescence, immunity, inflammation and tumorigenesis. Target genes of the upregulated miRNAs, including hsa-miR-125b-5p, hsa-miR-184, hsa-miR-24-3p, hsa-miR-205-5p and hsa-miR-376a-3p, are associated with cell proliferation. Target genes of downregulated hsa-miR-210 and hsa-miR-483-3p, are associated withcell proliferation. Targetgenes of downregulated miRNAs, including hsa-let-7b-3p, hsa-miR-106a, hsa-miR-203, hsa-miR-210, hsa-miR-23b-3p, hsa-miR-31, hsa-miR-328, hsa-miR-34a-3p, hsa-miR-574-3p and hsa-miR-720, are associated with cell differentiation. miRNAs associated with keratinocyte senescence included hsa-miR-130b-3p, hsa-miR-137, hsa-miR-138, hsa-miR-181a-5p, hsa-miR-191-3p, hsa-miR-34a-3p and hsa-miR-668. miRNAs associated with keratinocyte apoptosis included hsa-miR-205-5p and hsa-miR-184. miRNAs associated with keratinocyte migration included hsa-miR-21, hsa-miR-205-5p and hsa-miR-484-3p. miRNAs associated with skin pigmentation included hsa-miR-25 and hsa-miR434-5p. miRNAs associated with skin immune function and inflammation included hsa-miR-125b, hsa-miR-146a, hsa-miR-155, hsa-miR-203, hsa-miR-21, hsa-miR-221 and hsa-miR-222. miRNAs associated with skin tumor formation included dhsa-let-7b-3p, hsa-miR-125b-5p, hsa-miR-137, hsa-miR-182, hsa-miR-193b, hsa-miR-196a, hsa-miR-200b and hsa-miR-203. 
Table II. miRNAs upregulated $>5$-fold in epidermal stem cells compared with those in differentiated keratinocytes.

\begin{tabular}{lccc}
\hline & \multicolumn{2}{c}{ Initial signal } \\
\cline { 2 - 3 } miRNAs & 1638.40 & Epidermal stem cells & Absolute fold change $^{\mathrm{a}}$ \\
\cline { 2 - 4 } hsa-miR-197-5p & 18.91 & 32033.10 & 21.99 \\
hsa-miR-125b-5p & 129.74 & 334.35 & 19.89 \\
hsa-miR-376a-3p & 76.70 & 1986.32 & 17.22 \\
hsa-miR-345 & 10.13 & 1147.51 & 16.82 \\
hsa-miR-127-3p & 30.56 & 143.09 & 15.88 \\
hsa-miR-671-3p & 299.02 & 357.02 & 13.14 \\
hsa-miR-18a-5p & 89.15 & 3166.46 & 11.91 \\
hsa-miR-181c-5p & 446.64 & 910.34 & 11.48 \\
hsa-miR-222-5p & 4.17 & 3669.74 & 9.24 \\
hsa-miR-30d-3p & 661.46 & 33.00 & 8.89 \\
hsa-miR-374a-3p & 78.07 & 3808.10 & 6.47 \\
hsa-miR-545-3p & 349.03 & 5.03 \\
\hline
\end{tabular}



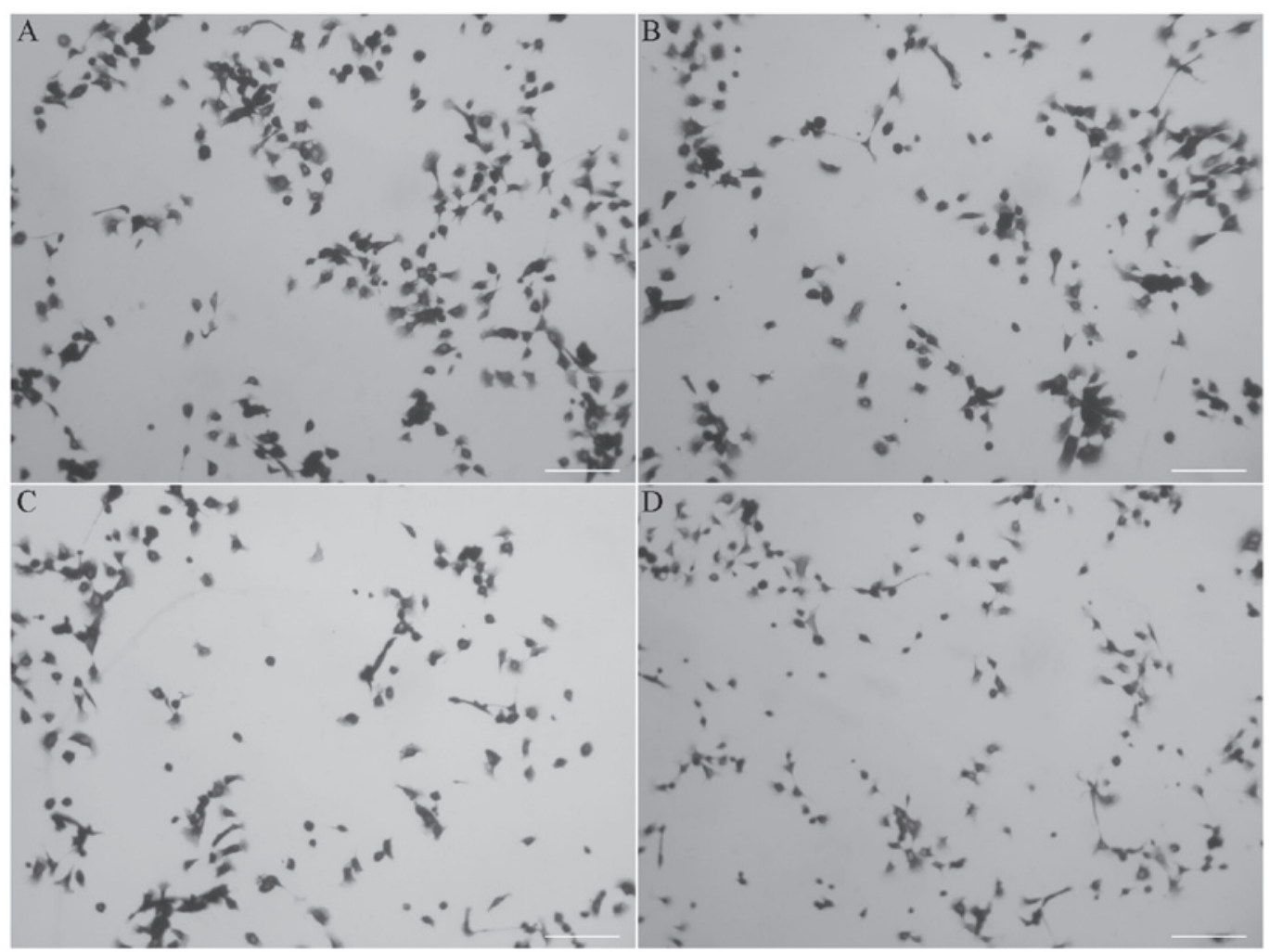

Figure 2. Immunocytochemical staining of human epidermal stem cells (ESCs) and differentiated keratinocytes (magnification, $\mathrm{x} 400$; scale bar, 20 $\mu \mathrm{m}$ ). (A) Positive expression of (A) CK19 and (B) integrin $\beta 1$ in human ESCs, and (C) CK1 and (D) CK10 in differentiated keratinocytes.

\section{Discussion}

miRNA genes are transcribed in the nucleus by RNA polymerase II or III into pri-miRNA, which is processed into pre-miRNA by Drosha and transported to the cytoplasm, where pre-miRNA is cleaved by Dicer into mature miRNA.
Mature miRNA selectively binds to the RNA-induced silencing complex, recognizes and binds to target genes in a complementary or incomplete complementary approach to either degrade target gene mRNA or inhibit its protein translation. Thus, this miRNA has functions in the post-transcriptional regulation of gene or protein expression 
Table III. miRNAs downregulated $>5$-fold in epidermal stem cells compared with those in differentiated keratinocytes.

\begin{tabular}{|c|c|c|c|}
\hline \multirow[b]{2}{*}{ miRNAs } & \multicolumn{2}{|c|}{ Initial signal } & \multirow[b]{2}{*}{ Absolute fold change ${ }^{a}$} \\
\hline & Differentiated keratinocytes & Epidermal stem cells & \\
\hline hsa-miR-203 & 2.86 & 0.10 & 25.45 \\
\hline hsa-miR-29b-3p & 20944.45 & 841.82 & 22.13 \\
\hline hsa-miR-34a-3p & 427.67 & 22.89 & 16.62 \\
\hline hsa-miR-574-5p & 154.53 & 12.12 & 11.33 \\
\hline hsa-miR-23b-3p & 1.14 & 0.10 & 10.17 \\
\hline hsa-miR-720 & 692.48 & 73.52 & 8.38 \\
\hline hsa-miR-20b-5p & 1008.73 & 132.81 & 6.75 \\
\hline hsa-miR-106a & 1495.80 & 259.09 & 5.13 \\
\hline
\end{tabular}

${ }^{a}$ Epidermal stem cells vs. differentiated keratinocytes.

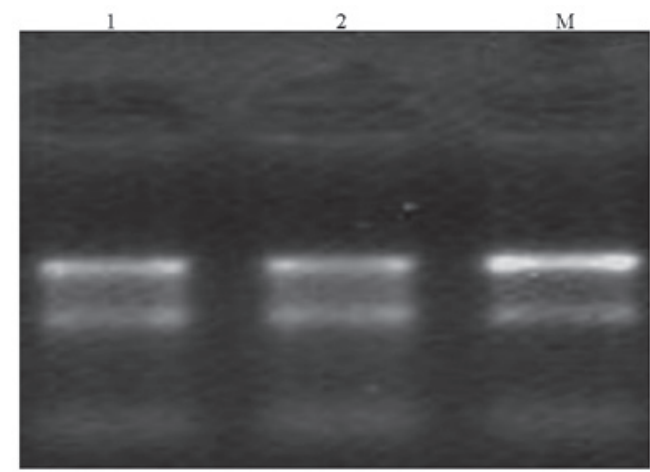

Figure 3. Quality control of total RNA. Lane 1, epidermal stem cells; lane 2, differentiated keratinocytes; M, HeLa cell RNA.

and achieves negative regulation of gene expression. miRNA genes only account for $1 \%$ of the total eukaryotic genes; however, target genes regulated by miRNA genes comprise $\sim 30 \%$ of the whole genome (10).

Skin morphogenesis is a complex process that has an important function in maintaining a stable miRNA regulatory network. This has been confirmed in numerous studies, and any abnormality in the expression of miRNAs induces skin morphogenesis disorder (11). ESCs are specific adult stem cells in skin tissue that are primarily located in the basal layer of the epidermis and in the distended section of the outer root sheath of hair follicles (12). Generally, ESCs perform asymmetric division, forming a stem cell with the same proliferation and differentiation potential and a transient amplifying cell (TAC). Through spinous and granular layers, TACs transform into terminally differentiated cells and enter the horny layer (3). While ESCs and differentiated keratinocytes are at different stages of the same developmental process, their biological features are markedly different, most likely due to differential miRNA expression (13). However, the differential expression, function and mechanism of miRNA in ESCs and differentiated keratinocytes remain unclear (14).

The present study applied microarray hybridization to comparably observe the differential miRNA expression profiles of ESCs and differentiated keratinocytes. The results showed 25 clearly upregulated miRNAs and 166 apparently downregulated miRNAs in ESCs. Target gene prediction demonstrated that miRNAs were closely correlated with cell proliferation, differentiation, immunity, inflammation, apoptosis and aging in the two cell groups. Yi et al (15) discovered that miR-200, miR-203 and miR-19/20 families are preferentially expressed in the epidermis. Sonkoly et al (16) analyzed the expression of miR-203 in healthy skin and other tissues. They observed that miR-203 expression in the skin is 100x higher than that in other tissues and thus named it as 'skin-specific miRNA'. The present research showed that in ESCs the downregulation of miR-203 was the most marked. Yi et al (17) reported that miR-203 promotes epidermal cell differentiation by controlling cell proliferation potential and inducing cell cycle arrest. Downregulation of miR-203 expression can inhibit cell differentiation, which indicates that miR-203 serves as a switch of proliferation and differentiation in skin development (i.e., miR-203 expression levels are low in proliferating cells and high in differentiating cells). Furthermore, high miR-203 expression inhibits proliferation and induces and promotes cell differentiation. miR-203 was shown to function at the post-transcriptional level, directly inhibiting the expression of target genes P63 (17) and SOCS-3 (16). Similar to that of miR-203, Let-7b expression was also shown to be downregulated in ESCs. Johnson et al (18) demonstrated that upregulation of Let-7 expression levels in human lung tissue interferes with $\mathrm{G}_{1} / \mathrm{S}$ transition and increases the number of cells at the $G_{0} / G_{1}$ phases by post-transcriptionally downregulating cell cycle control genes, such as CDK6, CDC25A and CCND, to inhibit cell proliferation, destroy cell multipotent properties and systematically induce cells into terminal differentiation. Similarly, miR-31 is downregulated in ESCs. Peng et al (19) found that miR-31 can activate the Notch pathway and promote stem cell differentiation though negative regulation of hypoxia-inducible factor (HIF)-1. Mardaryev et al (20) reported that miR-31 is highly expressed and involved in the hair growth cycle, preventing hair growth by inhibiting the target genes Krt16, Dlx3 and Fgf10. Conversely, inhibition of miR-31 expression increases the number of 
hair follicles and bulbs, as well as inducing hypertrophy of the outer root sheath of hair follicles. Furthermore, the expression levels of miR-210 in ESCs are downregulated by HIF-1 $\alpha$ and upregulated in hypoxic-ischemic wounds. This expression also negatively regulates the expression of cell cycle regulatory protein E2F3 to arrest cell cycle and thus inhibit cell proliferation (21). Similarly, miR-483-3p expression is downregulated in ESCs. Previous research has indicated that miR-483-3p is upregulated in the terminal phase of wound healing and inhibits the proliferation and migration of keratinocytes by suppressing the expression of the target genes MK2, MK167 and YAP1. Consequently, these functions lead to the termination of wound re-epithelization (22). Downregulated miR-137, miR-668 (23) and miR-191 (24) have been shown to correlate with replicative senescence of keratinocytes, which interferes with the $G_{1} / S$ phase transition in stem cells. Additionally, these miRNAs arrest the cell cycle, terminate proliferation and induce keratinocytes into the aging process by post-transcriptionally downregulating the cell cycle control genes SATB1 and CDK6.

The present study found that miR-125b was significantly upregulated in ESCs. Zhang et al (25) confirmed that miR-125b post-transcriptionally suppresses its target genes Blimp1 and VDR to maintain the multipotent properties of mammal keratinocytes, regulate self-renewal of stem cells and inhibit stem cell differentiation. The overexpression of miR-125b in ESCs and their progeny in transgenic mice results in extremely thick epidermis. In addition, upregulated miR-24 in ESCs was found to inhibit the expression of P27 and P16 proteins by post-transcriptionally suppressing cyclin-dependent kinase to promote the $G_{1} / S$ transition of stem cells, hence, overexpression of miR-24 promotes cell proliferation while antagonism of miR-24 results in the opposite (26). Upregulation of miR21-3p in ESCs is observed in wound healing and re-epithelization. TGF- $\beta 1$ upregulates the expression of miR-21, which post-transcriptionally suppresses TIMP3 and TIAM1, promoting keratinocyte migration and wound re-epithelization (27). Upregulated miR-205 inhibits target gene SHIP2 expression to promote keratinocyte migration and to resist keratinocyte apoptosis, which facilitates epithelium stability and wound healing. Antagonism of miR-205 can reduce the actin filaments within the wound, increase cell adhesion and enhance focal contact, thereby delaying wound healing (28).

In conclusion, microarray hybridization was performed to compare the differential miRNA expression profiles of ESCs and differentiated keratinocytes. A total of 25 upregulated miRNAs and 166 downregulated miRNAs were discovered. RT-qPCR results showed that the microarray hybridization technology was reliable. Target gene prediction demonstrated that miRNA target genes closely correlated with the levels of cell proliferation, differentiation, apoptosis and migration. These results facilitate the understanding of the processes and molecular pathways involved in normal skin development, as well as the screening of target genes that promote skin appendage development and wound healing. In addition, the results demonstrated that the promotion of keratinocyte dedifferentiation via interference with the associated miRNA may provide a novel source of seed cells for tissue-engineered skin.

\section{Acknowledgements}

This study was supported by grants from the National Natural Science Foundation of China (no. 81460293), the Science and Technology Planning Project of Jiangxi Province, China (no. 20133BBG70026) and the Scientific Research Project Foundation of Jiangxi Provincial Education Department (GJJ 12057).

\section{References}

1. Sandilands A, Sutherland C, Irvine AD and McLean WH: Filaggrin in the frontline: role in skin barrier function and disease. J Cell Sci 122: 1285-1294, 2009.

2. Sotiropoulou PA and Blanpain C: Development and homeostasis of the skin epidermis. Cold Spring Harb Perspect Biol 4: a008383, 2012.

3. Cangkrama M, Ting SB and Darido C: Stem cells behind the barrier. Int J Mol Sci 14: 13670-13686, 2013.

4. Moreno-Moya JM, Vilella F and Simón C: MicroRNA: key gene expression regulators. Fertil Steril 101: 1516-1523, 2014.

5. Cai Y, Yu X, Hu S and Yu J: A brief review on the mechanisms of miRNA regulation. Genomics Proteomics Bioinformatics 7: 147-154, 2009 .

6. Andl T, Murchison EP, Liu F, et al: The miRNA-processing enzyme dicer is essential for the morphogenesis and maintenance of hair follicles. Curr Biol 16: 1041-1049, 2006.

7. Yi R, Pasolli HA, Landthaler M, et al: DGCR8-dependent microRNA biogenesis is essential for skin development. Proc Natl Acad Sci USA 106: 498-502, 2009.

8. Kim DS, Cho HJ, Choi HR, Kwon SB and Park KC: Isolation of human epidermal stem cells by adherence and the reconstruction of skin equivalents. Cell Mol Life Sci 61: 2774-2781, 2004.

9. Schmittgen TD and Livak KJ: Analyzing real-time PCR data by the comparative C(T) method. Nat Protoc 3: 1101-1108, 2008

10. Lewis BP, Burge CB and Bartel DP: Conserved seed pairing, often flanked by adenosines, indicates that thousands of human genes are microRNA targets. Cell 120: 15-20, 2005.

11. Yi R and Fuchs E: MicroRNA-mediated control in the skin. Cell Death Differ 17: 229-235, 2010.

12. Ghadially R: 25 years of epidermal stem cell research. J Invest Dermatol 132: 797-810, 2012

13. Hildebrand J, Rütze M, Walz N, et al: A comprehensive analysis of microRNA expression during human keratinocyte differentiation in vitro and in vivo. J Invest Dermatol 131: 20-29, 2011.

14. Hildebrand J, Grundhoff A, Gallinat S, et al: MicroRNA profiling during human keratinocyte differentiation using a quantitative real-time PCR method. Methods Mol Biol 961: 193-200, 2013.

15. Yi R, O'Carroll D, Pasolli HA, et al: Morphogenesis in skin is governed by discrete sets of differentially expressed microRNAs. Nat Genet 38: 356-362, 2006.

16. Sonkoly E, Wei T, Janson PC, et al: MicroRNAs: novel regulators involved in the pathogenesis of psoriasis? PLoS One 2: e610, 2007.

17. Yi R, Poy MN, Stoffel M and Fuchs E: A skin microRNA promotes differentiation by repressing 'stemness'. Nature 452: 225-229, 2008.

18. Johnson CD, Esquela-Kerscher A, Stefani G, et al: The let-7 microRNA represses cell proliferation pathways in human cells. Cancer Res 67: 7713-7722, 2007.

19. Peng H, Kaplan N, Hamanaka RB, et al: microRNA-31/factor-inhibiting hypoxia-inducible factor 1 nexus regulates keratinocyte differentiation. Proc Natl Sci USA 109: 14030-14034, 2012.

20. Mardaryev AN, Ahmed MI, Vlahov NV, et al: Micro-RNA-31 controls hair cycle-associated changes in gene expression programs of the skin and hair follicle. FASEB J 24: 3869-3881, 2010.

21. Biswas S, Roy S, Banerjee J, et al: Hypoxia inducible microRNA 210 attenuates keratinocyte proliferation and impairs closure in a murine model of ischemic wounds. Proc Natl Acad Sci USA 107: 6976-6981, 2010.

22. Bertero T, Gastaldi C, Bourget-Ponzio I, et al: miR-483-3p controls proliferation in wounded epithelial cells. FASEB J 25: 3092-3105, 2011 
23. Shin KH, Pucar A, Kim RH, et al: Identification of senescence-inducing microRNAs in normal human keratinocytes. Int J Oncol 39: 1205-1211, 2011.

24. Lena AM, Mancini M, Rivetti di Val Cervo P, et al: MicroRNA-191 triggers keratinocytes senescence by SATB1 and CDK6 downregulation. Biochem Biophys Res Commun 423: 509-514, 2012.

25. Zhang L, Stokes N, Polak L and Fuchs E: Specific microRNAs are preferentially expressed by skin stem cells to balance self-renewal and early lineage commitment. Cell Stem Cell 8: 294-308, 2011
26. Giglio S, Cirombella R, Amodeo R, et al: MicroRNA miR-24 promotes cell proliferation by targeting the CDKs inhibitors p27Kip1 and p16INK4a. J Cell Physiol 228: 2015-2023, 2013.

27. Yang X, Wang J, Guo SL, et al: miR-21 promotes keratinocyte migration and re-epithelialization during wound healing. Int J Biol Sci 7: 685-690, 2011.

28. Yu J, Peng H, Ruan Q, et al: MicroRNA-205 promotes keratinocyte migration via the lipid phosphatase SHIP2. FASEB J 24: 3950-3959, 2010. 